STOMACH

\title{
Real time high resolution magnetic resonance imaging for the assessment of gastric motility disorders
}

\author{
W Ajaj, S C Goehde, N Papanikolaou, G Holtmann, S G Ruehm, J F Debatin, T C Lauenstein
}

Gut 2004;53:1256-1261. doi: 10.1136/gut.2003.038588

See end of article for authors' affiliations

Correspondence to:

Dr W Ajaj, Department for Diagnostic and

Interventional Radiology,

University Hospital Essen,

Hufelandstrasse 55,

D-45122 Essen,

Germany; waleed.ajaj@

uni-essen.de

Revised version received 4 February 2004

Accepted for publication

3 March 2004
Background: The aim of this study was to evaluate whether patients with increased or decreased gastric motility can be differentiated from healthy volunteers by means of real time magnetic resonance imaging (MRI).

Patients and methods: Ten healthy volunteers, 10 patients with gastroparesis, and 10 patients with functional pylorospasm/peptic pyloric stenosis underwent real time MRI. All patients were examined on two separate days; once prior to therapy and once after adequate therapy. Antral motility was quantified by calculating the gastric motility index.

Results: Patients with gastroparesis showed a lower motility index compared with the reference volunteer group while the mean motility index of the patient group with pylorospasm was more than three times higher than that of the reference value of the volunteer group. However, the gastric motility index in the patient group with gastroparesis increased, and in the group with functional pylorospasm/peptic pyloric stenosis it decreased significantly after therapy.

Conclusion: Real time MRI is a reliable tool for assessment of gastric motion. Furthermore, differences in gastric motility index in patients with increased or decreased gastric motility could be evaluated and quantified. Due to the non-invasive character of MRI, this imaging modality may be an attractive alternative to conventional invasive diagnostic tools for gastric motility disorders and therapeutic monitoring.
$\mathrm{T}$ raditional diseases of the gastrointestinal tract are based on the presence of structural lesions or biochemical abnormalities. ${ }^{12}$ However, disturbances in gastric motor function are believed to play a key role in the development of symptoms in patients with functional gastrointestinal disorders. Functional gastrointestinal disorders represent a major burden to society and to the affected patient. ${ }^{3}$ Based on the Rome Consensus, functional gastrointestinal disorders are now categorised into distinct entities based on symptom patterns. The most important examples of these disorders are the so-called irritable bowel syndrome, functional dyspepsia, and angina pectoris without detectable coronary ischaemia, referred to as syndrome $X$ or non-cardiac chest pain. ${ }^{5-8}$ In contrast, morphological diseases of the gastrointestinal tract are characterised by the presence of structural lesions such as stenosis or occlusion. ${ }^{10}$ While functional pathologies are highly prevalent, all available tests to assess motor function are either invasive, expose the patient to radiation, are somehow inaccurate, or cannot display gastric motility in real time imaging. ${ }^{11-13}$ Real time magnetic resonance imaging (MRI) has been proposed for evaluation of gastric motility. ${ }^{14-16}$ This modality combines the advantages of noninvasiveness and operator independence. Furthermore, MRI is not associated with ionising radiation exposure. Most MRI techniques have focused on evaluation of changes in gastric volume and/or morphology but with new real time sequences these limitations may be overcome. Mainly used for cardiac imaging, fast acquisition techniques may be applicable for evaluation of gastric motion and have been evaluated in volunteer trials. ${ }^{17}{ }^{18}$ To date, gastric motion has only been assessed in a few studies ${ }^{19}{ }^{20}$ which mainly assessed real time techniques in healthy volunteers.

The aim of this study was to determine the practicability of a real time fast imaging with steady state precession (TrueFISP) sequence for assessment of gastric motion in patients with gastric motility disorders and compare the results with reference values obtained in a healthy volunteer group.

\section{METHODS}

Ten healthy volunteers (group A: non-smokers, seven males and three females, mean age 37 years) with no history of gastrointestinal disease and 20 patients were examined. Ten patients suffered from gastroparesis (group B: six males and four females, mean age 55 years) and 10 patients had functional pylorospasm or peptic pyloric stenosis (group C: two males and eight females, mean age 34 years). The diagnosis was made based on clinical parameters (for example, gastroscopy, manometry). Patients were recruited over a nine months period. All patients with a clinical diagnosis of pathological gastric motility were consecutively asked if they would like to enter our study. The study was approved by the local institutional review board and written informed consent was obtained from each subject prior to examination.

The environmental temperature in the examination room was held constant at $20^{\circ} \mathrm{C}$ for all examinations. Sitting on the examination table just prior to commencing the examination, all subjects ingested $400 \mathrm{ml}$ of a high caloric commercially available vanilla pudding after a six hour fast (Ravensberger $100 \mathrm{kcal} / 100 \mathrm{~g}$ ). Preceding in vitro experiments had demonstrated that the signal intensity of the pudding on two dimensional TrueFISP images could be increased by adding a small amount of paramagnetic contrast in the form of gadopentetate dimeglumine (Gd-DTPA, Magnevist; Schering AG, Berlin, Germany) at a concentration of 1:200. Accordingly, $400 \mathrm{ml}$ of pudding were spiked with $2 \mathrm{ml}$ of Gd-DTPA prior to ingestion. To reduce air swallowing,

Abbreviations: MRI, magnetic resonance imaging; TrueFISP, fast imaging with steady state precession sequence; GMI, gastric motility index 
subjects were asked to ingest the pudding without haste over 7-10 minutes. Patients were examined once before therapy and then again after therapy (in group B, time between measurements was mean 5 (2)weeks; therapy comprised the motilin agonist erythromycin; in group $\mathrm{C}$, time between measurements was mean 7 (2) weeks; therapy comprised pylorotomy or endoscopic pyloric dilatation). Thus the two examinations took place on two different days.

MR examinations were performed on a $1.5 \mathrm{~T}$ scanner equipped with high performance gradients characterised by an amplitude of $40 \mathrm{mT} / \mathrm{m}$ and a slew rate of $200 \mathrm{mT} / \mathrm{m} / \mathrm{ms}$ (Magnetom Sonata; Siemens Medical Systems, Germany). For signal reception, a standard phased array body coil was used. Immediately following ingestion of the pudding, subjects were examined in the supine position. Subsequently, we scanned the first series after subjects were
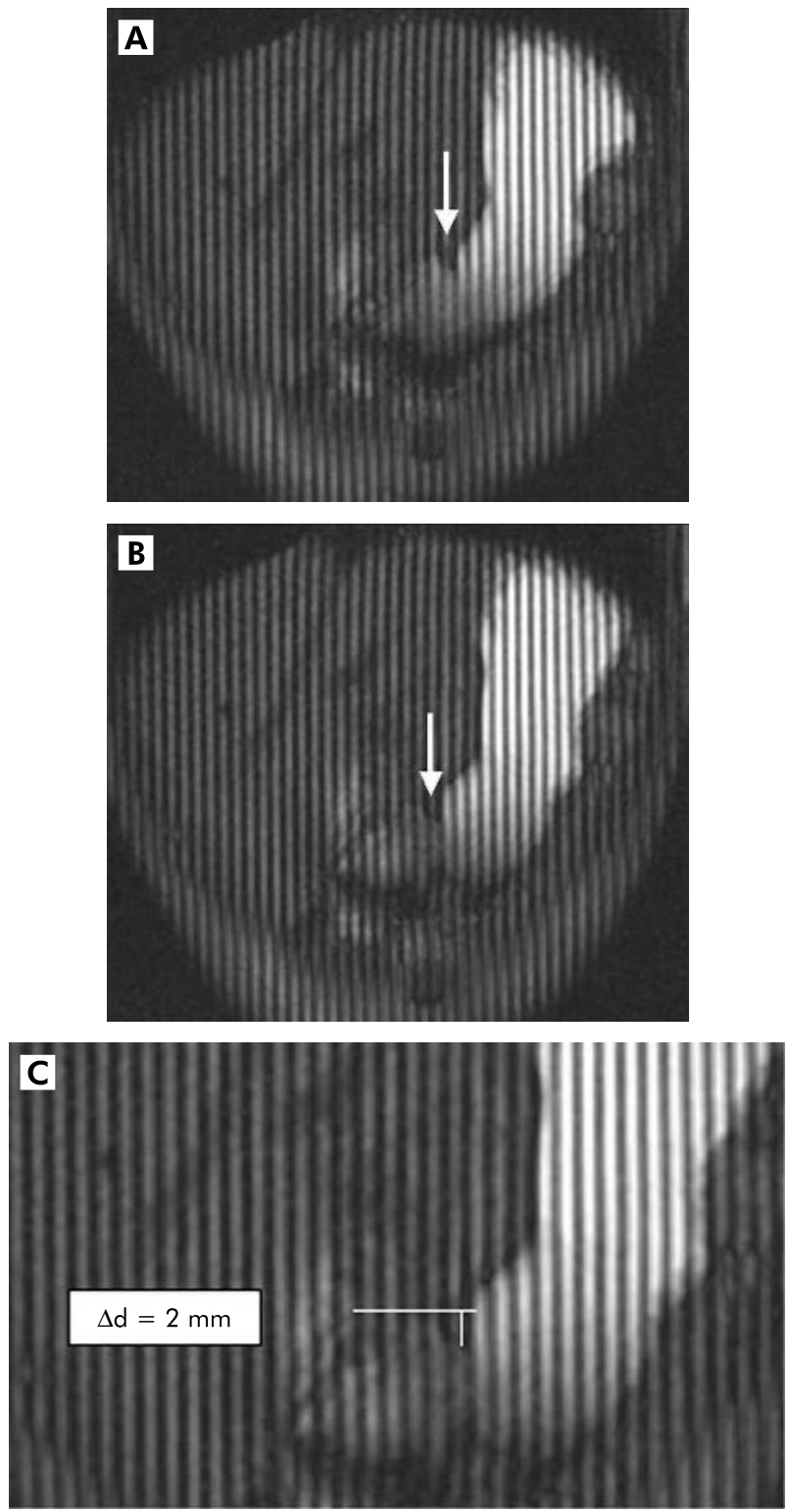

Figure 1 Two dimensional real time fast imaging with steady state precession sequence (TrueFISP) with tagging lines in an oblique plane displaying the antral axis. Images $(A)(t=0)$ and $(B)(t=20$ s) show the motion of the peristaltic wave towards the pylorus (arrow). In (C), the lowest amplitude of the according peristaltic wave is shown. Based on the distance that one wave passes within 20 seconds and its lowest amplitude, a motility index could be calculated. positioned on the table (mean 3 (1) minutes) at times 0,5 , $10,15,20,25$, and 30 minutes. To that end, a two dimensional real time TrueFISP sequence $(\mathrm{TR} / \mathrm{TE}=3.9$ / $1.9 \mathrm{~ms}$, flip $69^{\circ}$ ) was used with and without vertical tagging lines. Tagging lines were used to provide a reliable landmark for the spatial position of the stomach. The acquisition plane of the single $5 \mathrm{~mm}$ section was chosen interactively to be parallel to the axis of the gastric antrum using an axial localiser. This approach seemed feasible to us with respect to repositioning of the oblique coronal image plane in the following examinations on the same subject. The entire stomach was displayed. A $33 \times 40 \mathrm{~cm}$ field of view in conjunction with an acquisition matrix of $166 \times 256$ interpolated to $332 \times 512$ was chosen. Real time TrueFISP provided one image per second. Imaging was performed breath held over 20 seconds. For assessment of gastric motion, dark parallel tagging lines were applied in a craniocaudal direction in a similar manner to that described for cardiac MR imaging. ${ }^{16}$

All images were transferred to a post-processing workstation (Leonardo; Siemens Medical Systems, Erlangen, Germany) for subsequent qualitative and quantitative analysis.

For each real time data set a motility index was calculated. The frequency and propagation speed of gastric contraction were calculated. The travel distance $\Delta X$ was determined as the total distance the wave would propagate during a 20 second period. For this purpose, a free hand line was drawn along the gastric wall between the position of the wave at $0 \mathrm{~s}$ and at $20 \mathrm{~s}$. The software returns the curved distance. Gastric peristaltic wave velocity $(\mathrm{V})$ was defined as follows:

$$
V\left[\frac{m m}{s}\right]=\frac{\Delta X}{\Delta t}
$$

Subsequently, the gastric motility index (GMI) was determined. To this end, the respective deepness $\Delta d$ of the wave was determined by the difference in positions between the deepest point of the wave and the extrapolated gastric wall. Vertical tagging lines were not chosen for measuring the respective travel distance but as an indicator over potential movements of the stomach as a whole organ. If this were not controlled, false results with too large values for the distance of the single contraction would be obtained. No visible movement of the stomach was seen thus making the distance measurements more easy. To determine GMI, velocity $(\Delta V)$ was multiplied by the deepness of the contraction wave as it reaches the antrum $(\Delta d)$ (fig $1 \mathrm{~A}-\mathrm{C}$ ):

$$
\mathrm{GMI}\left[\frac{m m^{2}}{s}\right]=\Delta V \times \Delta d
$$

To determine all of these parameters, the coronal image was magnified by a factor of three. No additional software was used for these calculations; rather they were performed by the multiplanar reformation software which is a routine part of the workstation. Time needed for analysis of one patient's image data set, containing seven (at times 0, 5 10, $15,20,25$, and 30 minutes after the beginning of the examination) $\times 20$ images (one image per second) amounted to approximately 30 minutes.

Finally, data from all of the examinations were statistically analysed. The Wilcoxon rank test was used to determine the statistical significance of differences between gastric motility indices within each group. Differences between patients in groups $\mathrm{A}$ and $\mathrm{B}$ were compared with volunteers for each time point using the Mann-Whitney test. A Bonferroni correction 

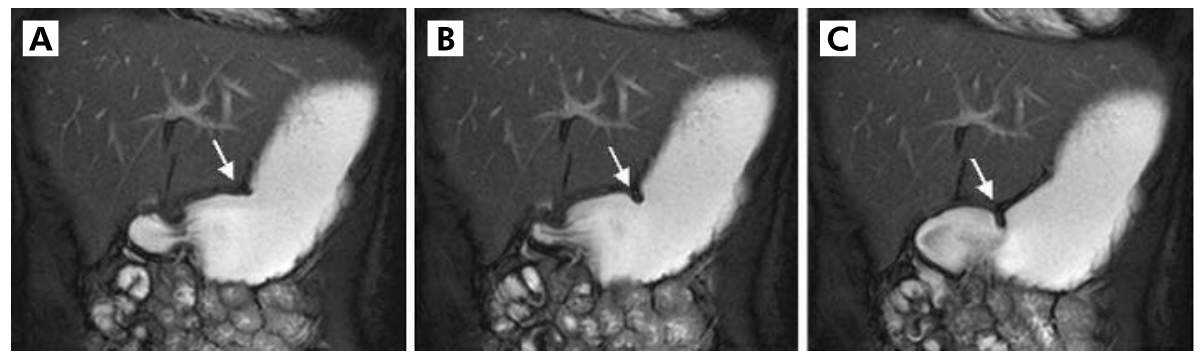

Figure 2 Two dimensional real time fast imaging with steady state precession sequence (TrueFISP) in an oblique plane displaying the antral axis. (A-C) Motion of the peristaltic wave towards the pylorus (arrow) in healthy volunteers. Based on the distance that one wave passes within 20 seconds and its lowest diameter, a motility index could be determined and gastric motion quantified.

for multiple comparisons was applied. A test value of $\mathrm{p}<0.05$ was considered to be statistically significant.

\section{RESULTS}

All subjects tolerated pudding ingestion well, and all MR examinations were completed without complications. Mean examination time, including pudding ingestion, was 40 minutes (range 38-43). The high caloric nutrient spiked with paramagnetic contrast was homogeneously bright on the real time two dimensional TrueFISP data sets. Thus delineation of the gastric lumen proved easy and robust. Results of examinations for volunteers (group A) were as follows: propagation speed of the antral wave increased between 0 and 15 minutes after ingestion of the pudding only slightly from a mean of $1.8 \mathrm{~mm} / \mathrm{s}$ to a mean of $2.4 \mathrm{~mm} / \mathrm{s}$, which remained unchanged from 15 to 30 minutes. Differences in propagation speed between time point 0 and all consecutive time points were statistically significant $(p<0.05)$. GMI, more sensitive to changes in gastric contraction, almost doubled, from a mean of 1.3 to $2.5 \mathrm{~mm}^{2} / \mathrm{s}$ over the first 15 minutes and continued to increase to $2.9 \mathrm{~mm}^{2} / \mathrm{s}$ at 25 and 30 minutes. Differences between time point 0 and all consecutive measurements proved to be statistically significant $(\mathrm{p}<0.05)$.

Patients with gastroparesis (group B) showed a dramatically reduced antral wave propagation speed as well as GMI over the 30 minutes of examination time compared with volunteers. While antral wave propagation speed increased from 0.6 to $1.0 \mathrm{~mm} / \mathrm{s}$ between 0 and 30 minutes after pudding ingestion $(\mathrm{p}<0.05)$, GMI increased from a very low mean value of 0.9 at 0 minutes to $1.7 \mathrm{~mm}^{2} / \mathrm{s}$ at 30 minutes $(\mathrm{p}<0.05)$. Mean GMI at 15 minutes was $1.5 \mathrm{~mm}^{2} / \mathrm{s}$ (range 0.9-1.9). However, 5 (2) weeks after therapy, mean GMI increased significantly and was $2.2 \mathrm{~mm}^{2} / \mathrm{s}$ (range 1.9-2.3) at the acquisition time point 15 minutes.

In contrast, patients with functional pylorospasm and peptic pyloric stenosis (group C) were found to have a mean motility index of $9 \mathrm{~mm}^{2} / \mathrm{s}$ at the acquisition time point 15 minutes (range 7-16) which decreased significantly after therapy ( 7 (2) weeks) to $6 \mathrm{~mm}^{2} / \mathrm{s}$ (range 5-9) at the acquisition time point 15 minutes. Figures 2-4 show an example of each study group. Mean gastric motility indices for the volunteer group as well as for both patient groups are shown in fig 5.
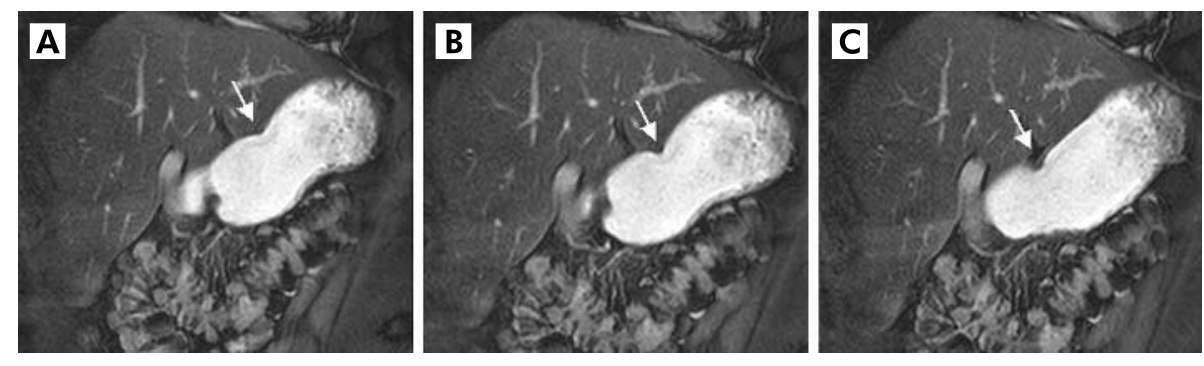

Figure 3 Two dimensional real time fast imaging with steady state precession sequence (TrueFISP) in an oblique plane displaying the antral axis. (A-C) Motion of the peristaltic wave towards the pylorus (arrow) in patients with decreased gastric motion. Based on the distance that one wave passes within 20 seconds and its lowest diameter, a motility index could be determined and gastric motion quantified.
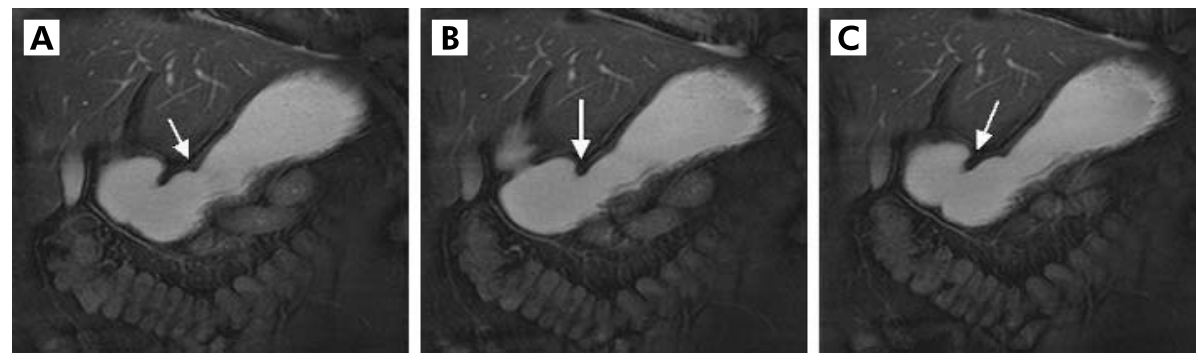

Figure 4 Two dimensional real time fast imaging with steady state precession sequence (TrueFISP) in an oblique plane displaying the antral axis. $(\mathrm{A}-\mathrm{C})$ Motion of the peristaltic wave towards the pylorus (arrow) in patients with increased gastric motility. Based on the distance that one wave passes within 20 seconds and its lowest diameter, a motility index could be determined and gastric motion quantified. 


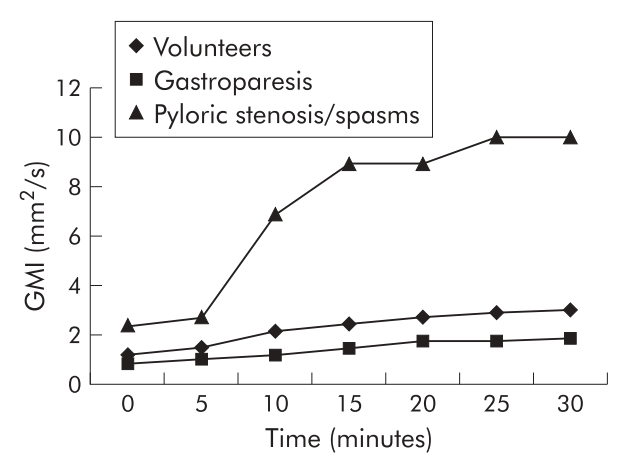

Figure 5 Mean gastric motility index GMl for the volunteer group and both patient groups prior to therapy. The motility index shows a statistically significant difference 15 minutes after pudding ingestion.

At all time points, GMI values of patients differed significantly from those of volunteers; this held true for patients in groups $\mathrm{B}$ and $\mathrm{C}$.

\section{DISCUSSION}

Gastric motility can be assessed by real time MRI. The acquired two dimensional real time TrueFISP data are robust. Patients with decreased gastric motion can be reliably distinguished from those with increased gastric motility. Due to the non-invasive nature of MRI, this imaging modality may be an attractive alternative to conventional invasive diagnostic tools for diagnosis of gastric motility disorders and consecutive therapeutic monitoring.

Despite the high prevalence of functional gastrointestinal pathologies, ${ }^{21}$ no single diagnostic test has emerged as generally recommended in a clinical environment. This observation reflects different drawbacks affecting each of the many tests in clinical practice. Thus gastric barostat studies assessing proximal motor function with an intragastric balloon provide accurate results ${ }^{11}$ but are hampered by their intrinsic invasiveness which translates into poor patient acceptance. On the other hand, electrogastrography, based on recording of gastric electrical activity from the body surface, is well accepted but fails to provide an acceptable correlation with gastric contractions. ${ }^{12}$ Finally, nuclear medicine studies are capable of quantifying gastric emptying but lack the spatial and temporal resolution to provide detailed data on gastric contraction and peristalsis. Furthermore, the test is associated with considerable exposure to ionising radiation. ${ }^{13}$

Lacking a technique with sufficient diagnostic accuracy and patient acceptance, these conditions frequently remain undiagnosed. ${ }^{1122}$ In view of the increasing availability of affordable and effective therapeutic strategies, ${ }^{23}{ }^{24}$ it is high time to overcome this diagnostic void. Respiratory motion artefacts in conjunction with susceptibility effects had for a long time prohibited the MR based analysis of the gastrointestinal tract. Reflecting recent hardware and software developments, MR imaging of the abdomen and consequently of the stomach has become possible by allowing acquisition of multislice or three dimensional data sets within a single breathhold. Advances in MRI have also led to increasing use of this technique in the evaluation of the gastrointestinal tract.

This initial technical report suggests that real time TrueFISP MR imaging can provide a non-invasive alternative to the present multimodality work up characterised by both sufficient diagnostic accuracy and high patient acceptance. Real time TrueFISP MR imaging is capable of depicting gastric motility with sufficient temporal and spatial resolution for a qualitative as well as quantitative assessment. The sequence permits excellent delineation of the stomach wall
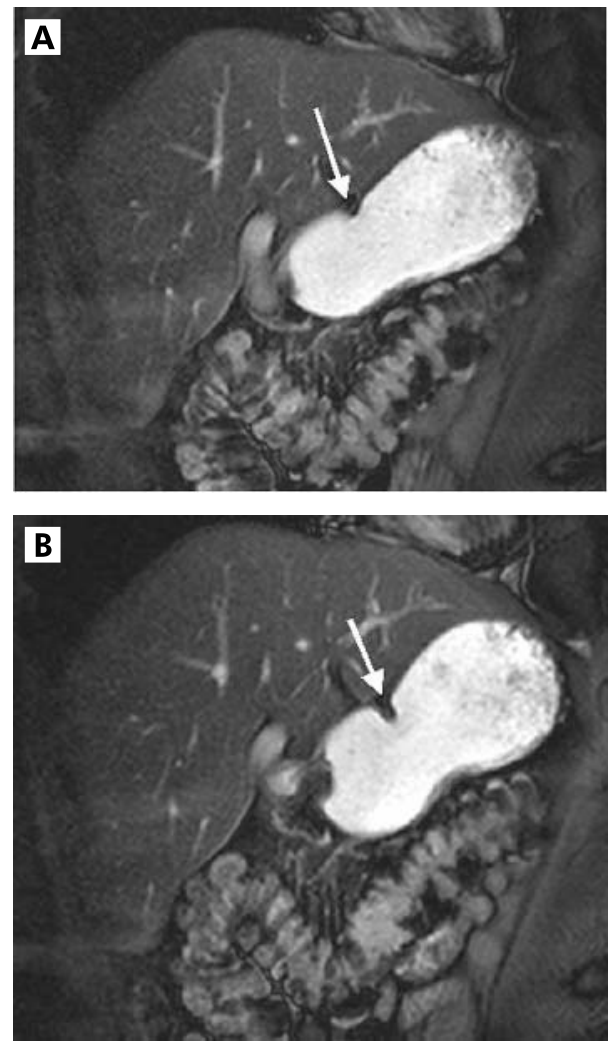

Figure 6 Two dimensional real time fast imaging with steady state precession sequence (TrueFISP) in a 51 year old female patient with diabetic gastroparesis. (A) Decreased contraction amplitude prior to therapy (arrow, gastric motility index (GMI) $1.2 \mathrm{~mm}^{2} / \mathrm{s}$ ). (B) However, the motilin agonist erythromycin caused an increase in contraction amplitude (arrow) six weeks after therapy. Subsequently, GMI increased to $1.8 \mathrm{~mm}^{2} / \mathrm{s}$.

from surrounding fat as well as the bright stomach lumen. ${ }^{25}{ }^{26}$ It is possible to use a solid meal for evaluation of gastric motility but the choice of a semisolid meal in our view seemed the most natural, ${ }^{27}$ and a semisolid meal can be homogenised better with gadolinium than a solid meal. Also, the total volume of the meal is the main factor for a prokinetic effect rather than the total caloric content; this was another reason for choosing the semiliquid meal. This approach has been shown to be reliable and reproducible. ${ }^{28}$ The high signal within the stomach in this sequence depends on paramagnetic contrast agent within the food ingested. In this study, we chose a pudding as the high caloric base for the oral contrast agent; in vitro experiments had revealed the pudding to be brightest following addition of a paramagnetic contrast in a concentration of 1:200.

Motivated by inherent non-invasiveness, operator independence, and lack of ionising radiation, MRI has in the past been proposed for evaluation of the stomach. Despite initial enthusiasm, the clinical utility of MRI for the detection and staging of gastric malignancies ${ }^{2-31}$ has remained rather limited. This reflects the lack of sufficient spatial resolution to depict the gastric mucosal surfaces. MR based analysis of functional gastric disorders on the other hand seems to have considerably more potential. ${ }^{14}{ }^{16-18} 3233$ First approaches proposed by Schwizer and colleagues ${ }^{34}$ were handicapped by long acquisition times rendering breathheld data acquisitions impossible. As a result, image quality was poor, translating into an imperfect correlation with nuclear scintigraphy. ${ }^{34}$ Despite consistent advances in MR technology, examination protocols remained cumbersome for some time. Thus Kunz 

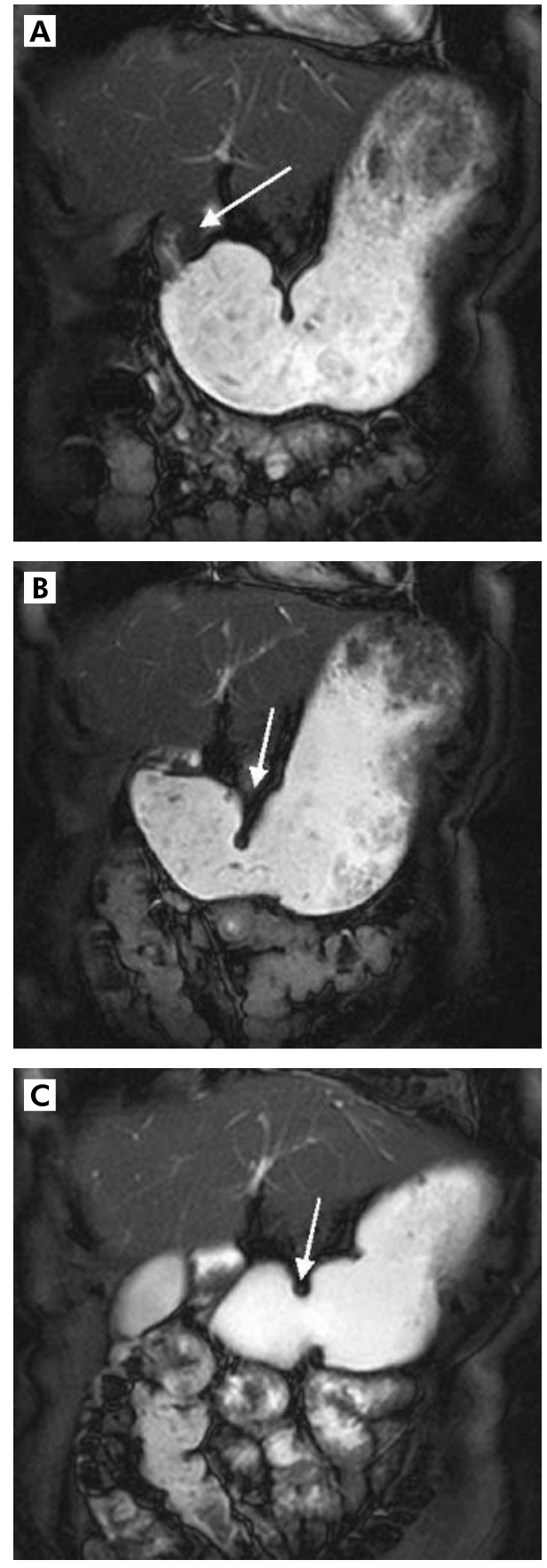

Figure 7 Two dimensional real time fast imaging with steady state precession sequence (TrueFISP) in a 33 year old female patients with peptic pyloric stenosis (A). Prior to therapy (B), motility index was $16 \mathrm{~mm}^{2} / \mathrm{s}$. After therapy (endoscopic pyloric dilatation (C)), motility index decreased to $9 \mathrm{~mm}^{2} / \mathrm{s}$.

and colleagues ${ }^{15} 16$ proposed assessment of gastric motor function based on MR acquisition lasting 60 seconds. To avoid respiratory motion artefacts, data collection was divided into four sections, during which volunteers were instructed to hold their breath. Differences in inspiratory depths limited volumetric accuracy.

In a volunteer study, administration of erythromycin resulted in substantial acceleration of gastric emptying. One of these studies also demonstrated the ability of time resolved three dimensional MRI to identify patients with gastric dyspepsia: three of six patients revealed much reduced gastric emptying rates in comparison with the mean determined in healthy volunteers. The real time imaging technique proposed in this study compliments the aforementioned efforts by providing a real time analysis of gastric motion. The peristaltic wave itself can be resolved with both sufficient temporal and spatial resolution to permit both a qualitative and quantitative assessment. As the time needed for calculation of the respective values is approximately $30 \mathrm{~min}$ utes, this can be performed in a routine clinical setting but would only be used in dedicated cases. Accordingly, both peristaltic wave velocity as well as GMI could be readily determined. However, real time sequences are not appropriate for assessment of gastric emptying. For this, the protocol could be amplified with three dimensional sequences which have been shown to sufficiently display gastric volumes and enable the calculation of gastric emptying rates. MR has the advantage over conventional radiography that volumetric measurements can be performed; thus morphological and functional information can be gained with a single method. In the future, additional information on the gastric wall might be gained by high resolution images (for example, if parallel acquisition techniques are used).

Based on tagging techniques, gastric peristaltic motion can be objectively quantified and hence characterised. Three easily measurable parameters permit determination of both peristaltic wave velocity as well as GMI. Based on these parameters, the GMI of the stomach, the pharmacological effect of the motility-modifying substance erythromycin, the effect of surgery (pylorotomy), and the effect of endoscopic pyloric dilatation on gastric peristalsis were successfully illustrated. Furthermore, the clinical impact of the technique is characterised by its non-invasive nature and lack of exposure to ionising radiation. Based on these observations, oral therapy with the motilin agonist erythromycin was shown to cause significant acceleration of antral peristaltic waves and an increase in GMI (fig 6A, B) whereas surgery and pyloric dilatation caused a significant decrease in GMI (fig $7 \mathrm{~A}-\mathrm{C}$ ).

We conclude that real time TrueFISP imaging is feasible for assessment of gastric motility and has the potential to diagnose gastric functional disease on the basis of GMI measurements. The acquired two dimensional real time TrueFISP data are robust and characterised by sufficient temporal and spatial resolution.

\section{Authors' affiliations}

W Ajaj, S C Goehde, N Papanikolaou, G Holtmann, S G Ruehm, J F Debatin, T C Lauenstein, Department for Diagnostic and Interventional Radiology, University Hospital Essen, Essen, Germany

\section{REFERENCES}

1 Neal KR, Hebden J, Spiller R. Prevalence of gastrointestinal symptoms six months after bacterial gastroenteritis and risk factors for development of the irritable bowel syndrome: postal survey of patients. BMJ 1997;314:779-82

2 Holtmann G, Talley NJ, Mitchell H, et al. Antibody response to specific $\mathrm{H}$. pylori antigens in functional dyspepsia, duodenal ulcer disease, and health. Am J Gastroenterol 1998;93:1222-7.

3 Haukim N, Bidwell JL, Smith AJ, et al. Cytokine gene polymorphism in human disease: on-line databases, supplement 2. Genes Immun 2002;3:313-30.

4 Kruger R, Hardt C, Tschentscher F, et al. Genetic analysis of immunomodulating factors in sporadic Parkinson's disease. I Neural Transm 2000;107:553-62.

5 Holtmann G, Gschossmann J, Neufang-Huber J, et al. Differences in gastric mechanosensory function after repeated ramp distensions in non-consulters with dyspepsia and healthy controls. Gut 2000;47:332-6.

6 Holtmann G, Gschossmann J, Holtmann M, et al. H. pylori and functional dyspepsia: increased serum antibodies as an independent risk factor? Dig Dis Sci 2001;46:1550-7.

7 Holtmann G, Talley NJ, Goebell H. Association between H. pylori, duodenal mechanosensory thresholds and small intestinal motility in chronic unexplained dyspepsia. Dig Dis Sci 1996;41:1285-91. 
8 Kunstmann E, Hardt C, Crabtree JE, et al. Helicobacter pylori infection: CagAspecific antibodies are associated with clinical outcome, but not with HLA-class II polymorphisms of the host. Int J Med Microbiol 2003;292:537-40.

9 Cerwenka H, Bacher H, Mischinger HJ. Pyloric obstruction caused by prolapse of a hyperplastic gastric polyp. Hepatogastroenterology 2002:49:958-60.

10 Hagiwara A, Sonoyama Y, Togawa T, et al. Combined use of electrosurgical incisions and balloon dilatation for the treatment of refractory postoperative pyloric stenosis. Gastrointest Endosc 2001;53:504-8.

11 Sarnelli G, Vos R, Cuomo R, et al. Reproducibility of gastric barostat studies in healthy controls and in dyspeptic patients. Am J Gastroenterol 2001;96:1047-53.

12 Sanmiguel CP, Mintchev MP, Bowes KL. Electrogastrography: a noninvasive technique to evaluate gastric electrical activity. Can J Gastroenterol 1998; 12:423-30.

13 Zahn A, Langhans CD, Hoffner S, et al. Measurement of gastric emptying by 13C-octanoic acid breath test versus scintigraphy in diabetics. Z Gastroenterol 2003;41:383-90.

14 Marciani L, Young P, Wright J, et al. Echoplanar imaging in GI clinical practice: assessment of gastric emptying and antral motility in four patients. J Magn Reson Imaging 2000;12:343-6.

15 Kunz P, Crelier GR, Schwizer W, et al. Gastric emptying and motility: assessment with MR imaging-preliminary observations. Radiology 1998;207:33-40.

16 Kunz P, Feinle C, Schwitzer W, et al. Assessment of gastric motor function during the emptying of solid and liquid meals in humans by MRI. Magen Reson Imaging 1999;9:75-80.

17 Quick HH, Kuehl H, Kaiser G, et al. Interventional MRA using actively visualized catheters, TrueFISP, and real-time image fusion. Magn Reson Med 2003:49:129-37.

18 Kramer U, Fenchel M, Helber U, et al. Multislice TrueFISP-MR imaging for identifying stress-induced myocardial functional disturbances in coronary heart disease. Rofo Fortschr Geb Rontgenstr Neven Bildgeb Verfahr 2003;175:1355-62.

19 Indiireshkumar K, Brasseur JG, Faas $\mathrm{H}$, et al. Relative contributions of "pressure pump" and "peristaltic pump" to gastric emptying. Am J Physiol Gastrointest Liver Physiol 2000;278:G604-16.
20 de Zwart IM, Mearadji B, Lamb HJ, et al. Gastric motility: comparison of assessment with real-time MR imaging or barostat measurement initial experience. Radiology 2002;224:592-7.

21 Richter JE. Dyspepsia: organic causes and differential characteristics from functional dyspepsia. Scand J Gastroenterol Suppl 1991;182:11-16.

22 Maughan RJ, Leiper JB. Methods for the assessment of gastric emptying in humans: an overview. Diabet Med 1996;13:6-10.

23 Quigley EM. Pharmacotherapy of gastroparesis. Expert Opin Pharmacother 2000;1:881-7.

24 Hall JA, Washabau RJ. Diagnosis and treatment of gastric motility disorders. Vet Clin North Am Small Anim Pract 1999;29:377-95.

25 Scheffler K, Hennig J. Is TrueFISP a gradient-echo or a spin-echo sequence? Magn Reson Med 2003;49:395-7.

26 Herborn CU, Vogt F, Lauenstein TC, et al. MRI of the liver: can True FISP replace HASTE? J Magn Reson Imaging 2003;17:190-6.

27 Hawighorst H, Schoenberg SO, Knopp MV, et al. Hepatic lesions: morphologic and functional characterization with multiphase breath-hold 3D gadolinium-enhanced MR angiography-initial results. Radiology 1999:210:89-96.

28 Marciani L, Young P, Wright J, et al. Antral motility measurements by magnetic resonance imaging. Neurogastroenterol Motil 2001;13:511-18.

29 Kim AY, Han JK, Seong CK, et al. MRI in staging advanced gastric cancer: is it useful compared with spiral CT? J Comput Assist Tomogr 2000;24:389-94.

30 Sohn KM, Lee JM, Lee SY, et al. Comparing MR imaging and CT in the staging of gastric carcinoma. AJR 2000;174:1551-7.

31 Schmid MR, Hany TF, Knesplova L, et al. 3D MR gastrography: exoscopic and endoscopic analysis of the stomach. Eur Radiol 1999;9:73-7.

32 Ploutz-Snyder L, Foley J, Ploutz-Snyder R, et al. Gastric gas and fluid emptying assessed by magnetic resonance imaging. Eur J Appl Physiol Occup Physiol 1999;79:212-20.

33 Bilecen D, Scheffler K, Seifritz E, et al. Hydro-MRI for the visualization of gastric wall motility using RARE magnetic resonance imaging sequences. Abdom Imaging 2000;25:30-4.

34 Schwizer W, Maecke H, Fried M. Measurement of gastric emptying by magnetic resonance imaging in humans. Gastroenterology 1992; 103:369-76.

\section{EDITOR'S QUIZ: GI SNAPSHOT}

\section{A rare cause of abdominal pain}

\section{Clinical presentation}

A female, aged 22 years, with alopecia presented with acute onset left upper quadrant abdominal pain and hypotension (blood pressure 80/40). There was no history of trauma. Abdominal examination demonstrated guarding in the left upper quadrant and urgent blood investigations revealed neutrophil leucocytosis with a normal serum amylase. There was no free air under the diaphragm on an erect chest $x$ ray and an abdominal radiograph was unremarkable. In view of the unexplained localised peritonitis, an abdominal computed tomography (CT) scan was requested.

\section{Question}

What conclusions can you draw from this CT scan? See page 1266 for answer

This case is submitted by:

W H Chan, I Zerizer

Emergency Department, St Thomas Hospital, London, UK N Shergill, I Shergill, S Dougan

Department of General Surgery, Watford General Hospital, Watford, UK

Correspondence to: Dr W H Chan, Emergency Department, St Thomas' Hospital, Lambeth Palace Rd, London SEI 7EP, UK waibruceleechan@hotmail.com
Robin Spiller, Editor

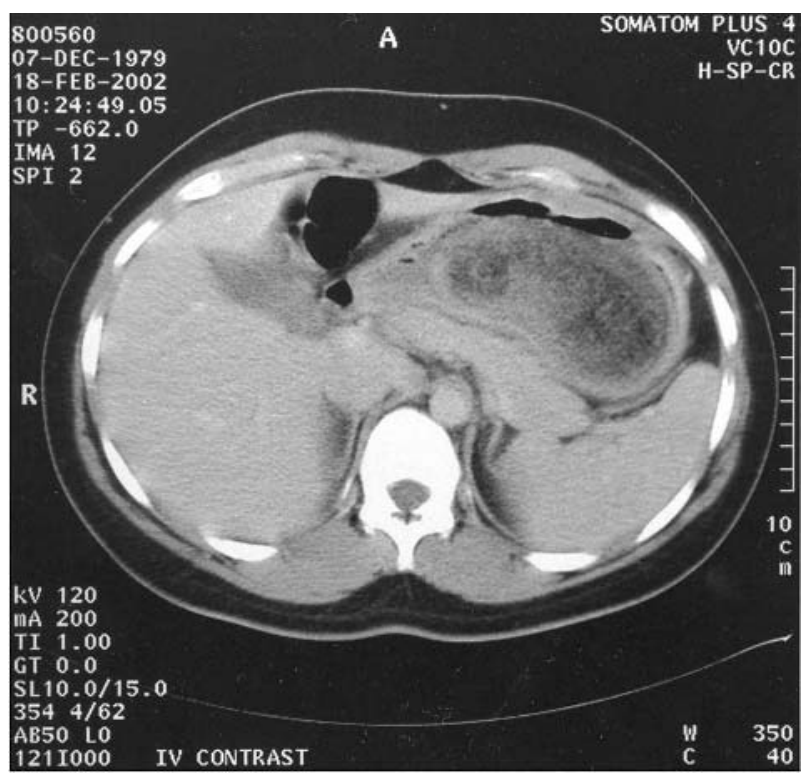

Figure 1 Abdominal computed tomography. 